\title{
A extensão universitária e sua relação com a qualidade de vida de idosos vulneráveis
}

\section{amazônidas}

\author{
University extension and its relationship with the quality of life of vulnerable elderly amazonians
}

La extensión universitaria y su relación con la calidad de vida de adultos mayores vulnerables amazónicos

Daiane de Souza Fernandes

ORCID: https://orcid.org/0000-0001-6629-4222

Universidade Federal do Pará, Brasil E-mail: daissf@yahoo.com.br

Julielen Larissa Alexandrino Moraes ORCID: https://orcid.org/0000-0003-3753-6645 Universidade Federal do Pará, Brasil

E-mail: julielen.alexandrino@yahoo.com

Gabriel de Luca Sousa Bandeira ORCID: https://orcid.org/0000-0001-9607-2348 Universidade Federal do Pará, Brasil E-mail: gabrielband9@gmail.com

Wanderson Santiago de Azevedo Junior ORCID: https://orcid.org/0000-0001-6125-3261 Universidade Federal do Pará, Brasil E-mail: wanderson_santiago12@hotmail.com Thamyres Batista Procópio ORCID: https://orcid.org/0000-0002-1300-9915 Universidade Federal do Pará, Brasil E-mail: thamyresprocopio@gmail.com Elizabeth França de Freitas ORCID: https://orcid.org/0000-0002-8402-0732 Universidade Federal do Pará, Brasil E-mail: elizabeth.enf80@gmail.com

Lucia Hisako Takase Gonçalves ORCID: https://orcid.org/0000-0001-5172-7814 Universidade Federal do Pará, Brasil E-mail: lhtakase@gmail.com

\begin{abstract}
Resumo
Objetivo: relatar a experiência de atividades desenvolvidas em um projeto de extensão universitário com idosos, onde foram empregadas metodologias participativas com valorização de questões sociais e culturais dos envolvidos. Metodologia: Trata-se de um estudo de abordagem qualitativa, descritiva, do tipo relato de experiência. Este relato de experiência foi elaborado a partir das vivências do projeto de extensão universitária "Idoso Saudável", vinculado a atividade curricular Atenção Integral a Saúde do Adulto e Idoso do terceiro período do curso de Enfermagem da Universidade Federal do Pará (UFPA) Resultado: O desenvolvimento de atividades com idosos é primordial para o estimular o envelhecimento ativo e com qualidade de vida, tal que se incrementa o processo de formação profissional em gerontogeriatria, uma vez que participam do projeto, os estudantes de Enfermagem. Considerações finais: Atividades extensionistas são um importante elo entre a universidade e comunidades. Observou-se o impacto na qualidade de vida dos idosos participantes, bem como na visão mais ampliada e especializada do processo de envelhecimento, além da importancia de fortalecimento de estudos na área para efetivar o protagonismo e impacto na população idosa.
\end{abstract}

Palavras-chave: Extensão universitária; Ações extensionistas; Idosos; Envelhecimento ativo; Qualidade de vida.

\begin{abstract}
Objective: to report the experience of activities carried out in a university extension project with the elderly, where participatory methodologies were used to value the social and cultural issues of those involved. Methodology: This is a qualitative, descriptive study, an experience report type. This experience report was elaborated from the experiences of the university extension project "Healthy Elderly", linked to the curricular activity Integral Attention to Adult and Elderly Health of the third period of the Nursing course at the Federal University of Pará (UFPA) Result: The the
\end{abstract}


development of activities with the elderly is essential to stimulate active aging and with quality of life, such that the process of professional training in gerontogeriatrics is increased, since nursing students participate in the project. Final considerations: Extension activities are an important link between the university and communities. The impact on the quality of life of the elderly participants was observed, as well as on the broader and more specialized view of the aging process, in addition to the importance of strengthening studies in the area to effect the protagonism and impact on the elderly population.

Keywords: University extension; Extension actions; Seniors; Active aging; Life quality.

\section{Resumen}

Objetivo: dar a conocer la experiencia de las actividades desarrolladas en un proyecto de extensión universitaria con personas mayores, donde se utilizaron metodologías participativas con la valoración de la problemática social y cultural de los involucrados. Metodología: Se trata de un estudio cualitativo, descriptivo, tipo relato de experiencia. Este relato de experiencia se elaboró a partir de las vivencias del proyecto de extensión universitaria "Anciano Saludable", vinculado a la actividad curricular Atención Integral a la Salud de Adultos y Ancianos del tercer período del curso de Enfermería de la Universidad Federal de Pará (UFPA) Resultado: La el desarrollo de actividades con las personas mayores es fundamental para estimular el envejecimiento activo y con calidad de vida, de manera que se incremente el proceso de formación profesional en gerontogeriatría, ya que en el proyecto participan estudiantes de enfermería. Consideraciones finales: Las actividades de extensión son un vínculo importante entre la universidad y las comunidades. Se observó el impacto en la calidad de vida de los ancianos participantes, así como en la visión más amplia y especializada del proceso de envejecimiento, además de la importancia de fortalecer los estudios en el área para incidir en el protagonismo e impacto en la población anciana.

Palabras clave: Extensión universitaria; Acciones de extensión; Personas mayores; Envejecimiento activo; Calidad de vida.

\section{Introdução}

O envelhecimento humano pode ser interpretado como um processo multidimensional, que engloba mudanças dependentes de fatores biológicos, psicológicos e sócio-históricos. Essas nuances incluem as singularidades de cada indivíduo, como genética, estilo de vida, comuns a certos grupos populacionais a exemplo daqueles com características socioeconômicas, histórico-culturais próprios (Martins et al. 2019).

Atualmente está ocorrendo uma mudança expressiva na estrutura etária das populações, com um aumento significativo da expectativa de vida. Alguns fatores impulsionaram esta mudança, como a redução da natalidade, avanços da medicina, investimentos em saúde pública e questões socioeconômicas.

De acordo com dados demográficos, em 2019 havia 703 milhões de pessoas com 65 anos ou mais na população global, no mais há expectativa de que este número possa dobrar seu valor nos próximos 30 anos. Ademais, a mulher se destaca em longevidade com expectativa de vida de 74,7 anos, superando em até 5 anos, o homem, com 69,9 anos (Organização da Nações Unidas ONU, 2020).

No Brasil, a população passa por um processo de envelhecimento rápido, devido principalmente pela significativa redução da taxa de fecundidade e aumento da longevidade. De 1960 para 2010, a expectativa de vida ao nascer aumentou em 25 anos. Em 2015, a estimativa da expectativa de vida ao nascer para as mulheres foi de 79,1 anos, e para os homens de 71,9 anos (Freire et al., 2017; Instituto Brasileiro de Geografia e Estatísticas (IBGE, 2016). Permeando tais projeções, em 2030 haverá um contingente de 32 milhões $(18,8 \%)$, dado que torna o Brasil a sexta maior população de idosos do mundo e por volta de 2050, essa porcentagem alcançará os 30\%, de modo que o total de idosos em idade maior ou igual a 80 anos será maior que o de crianças menores de quatro anos (Medeiros et al., 2015; Martins et al., 2019).

A qualidade de vida pode ser definida como a avaliação multidimensional e subjetiva, referenciada a critérios socionormativos e intrapessoais, a respeito das relações atuais, passadas e prospectivas entre o indivíduo e o seu ambiente (Mallmann et al., 2015).

Em um contraponto com a velhice, a qualidade de vida está relacionada à capacidade de adaptar-se às perdas físicas, sociais e emocionais, à situação socioeconômica, ao amparo familiar, à manutenção da atividade intelectual, diminuição na 
participação de atividades que exigem maior mobilidade devido perda de força e massa muscular ou obesidade, ao nível de escolaridade, em suma, à valorização dos aspectos favoráveis da vida em sua totalidade (Bergman, 2020; Bodner, 2019; Alves et al., 2020; Tessari et al., 2016; Morley \& Malmstrom, 2013).

Com isso, grupos de convivência para pessoas idosas surgem como uma contribuição para o usufruto do envelhecimento ativo, saudável e com mais qualidade de vida. A participação de idosos nesses grupos pode trazer diversos benefícios, tais como: afastar a solidão, propiciar amizades, aumentar a autoestima, melhorar a integração com os familiares, resgatar valores pessoais e sociais, promover suporte social e a adoção de estilo de vida mais saudável pautado na política de envelhecimento ativo, uma vez que nesses grupos são realizadas atividades culturais, de lazer, intelectuais, físicas, manuais, artísticas e de convívio geral (Freire et al., 2017; Pitz \& Matsuchita, 2015).

Dentro da educação, essas formações grupais inserem a pessoa idosa em um ambiente educativo e transformador, levando o idoso ser a figura principal no processo de vínculos e saberes distintos (Benedetti et al., 2012; Freire et al., 2017).

As ações de educação em saúde voltadas aos idosos tornam-se ferramentas eficazes para que essas pessoas desenvolvam a capacidade de intervenção sobre suas próprias vidas e sobre o ambiente com o qual interagem e assim, criarem condições para se apropriarem de sua própria existência. Essas ações devem ser ampliadas e desenvolvidas em todos os seguimentos da população idosa, segundo suas necessidades e perspectivas (Santos et al., 2014).

No contexto educacional, a Extensão Universitária se destaca por configurar uma estratégia que favorece o processo de ensino-aprendizagem, o qual articula saberes que envolvem o meio acadêmico, científico e comunitário, colaborando para a formação de cidadãos com variadas formas de pensar e agir na vivência da saúde (Silva et al., 2017).

Dentro dessa perspectiva, há a necessidade e importância de agir em prol da população idosa, permeando os saberes do meio acadêmico, na utilização e difusão de um dos tripés da universidade, a extensão universitária, destacando o ser enfermeiro como educador em saúde na comunidade.

Este artigo tem o objetivo de relatar a experiência dos autores do projeto de extensão universitária com idosos vulneráveis vivendo em comunidade carente, aos quais foram desenvolvidas metodologias participativas com valorização de suas questões sociais e culturais, com vistas à promoção e manutenção de qualidade de vida.

\section{Metodologia}

Trata-se de um estudo de abordagem qualitativa, descritiva, do tipo relato de experiência. Nesse método tem-se a importância da interpretação e opinião do pesquisador sob dado fenômeno e consequentemente seus impactos na comunidade em geral. O processo é mais focado no percurso dessas descrições e as respostas e reações dos participantes delimita o foco da pesquisa como um todo (Pereira et al., 2018).

Este relato de experiência foi elaborado a partir das vivências do projeto de extensão universitária "Idoso Saudável", vinculado a atividade curricular Atenção Integral a Saúde do Adulto e Idoso do terceiro período do curso de Enfermagem da Universidade Federal do Pará (UFPA), administrado por docentes da Faculdade de Enfermagem, estudantes de pós-graduação (modalidade Residência em Saúde do Idoso) e estudantes da graduação, totalizando 15 colaboradores, tendo como público alvo pessoas com idade igual ou superior a 60 anos vinculados ao Centro de Referência em Assistência Social (CRAS) de um bairro de periferia circunvizinho ao campus da UFPA, em Belém - Pará. O projeto deu início em 2014 e sua manutenção tem caráter permanente.

Ao caracterizar os participantes do projeto, por idade e sexo, observou-se um grupo etário de 60 a 86 anos, sendo composto em sua maioria por idosos do sexo feminino. Participavam algumas pessoas com menos de 60 anos, estes, por vezes, são filhos e acompanhantes dos idosos ou que demonstraram interesse em participar das atividades. 
As atividades de educação em saúde realizadas com o grupo utilizavam atividades lúdicas, onde os extensionistas elaboravam encontros com dinâmicas de cunho educativo, baseadas nas demandas manifestadas pelos idosos participantes, com o intuito sempre de estimular a autonomia e promover a observância dos preceitos do envelhecimento ativo. Os encontros mensais ocorriam no ambiente do CRAS, em três momentos.

\subsection{Primeiro Momento}

O início da reunião era dedicado a recepção e acolhimento dos idosos, bem como o cadastro dos que estavam participando pela primeira vez no projeto. Realizava-se uma dinâmica inicial, do tipo "quebra-gelo", com o intuito de possibilitar um momento de integração entre os participantes, descontração e fortalecimento de vínculo entre eles.

\subsection{Segundo momento}

Destinado a apresentação e explanação da temática, de forma interdisciplinar, com enfoque no desenvolvimento do envelhecimento ativo, com temas diferentes a cada mês, voltados para questões de cidadania, prevenção de doenças e morbidades, hábitos de vida saudáveis, valorização e empoderamento da pessoa idosa. Os assuntos eram abordados por meio de uma linguagem simples e de fácil compreensão, através de metodologias ativas com recursos audiovisuais, buscando viabilizar um maior entendimento sobre os temas abordados e estimular o compartilhamento de saberes prévios, experiências e opiniões a respeito do tema.

\subsection{Terceiro Momento}

Após a explanação do tema a ação educativa era finalizada com uma dinâmica de fixação do assunto abordado, utilizando estratégias lúdicas que estimulassem a participação e interação com os idosos como jogos, gincanas, oficinas, entre outras atividades. Dessa forma era possível verificar se os objetivos da ação foram alcançados.

\section{Resultados e Discussão}

\subsection{Atividades extensionistas como estratégia de qualidade de vida para idosos}

A Organização Mundial da Saúde (OMS) define que: "A Qualidade de Vida é a percepção de bem-estar de uma pessoa, que deriva de sua avaliação do quanto realizou daquilo que idealiza como importante para uma boa vida e de seu grau de satisfação com o que foi possível concretizar até aquele momento" (Silva et al., 2017; Martins et al., 2019).

Ratifica-se a necessidade de educação em saúde com idosos a respeito de seus hábitos, qualidades de vida e suas morbidades, tendo em vista que a OMS (2020) informa que as doenças crônicas não transmissíveis são as maiores causas de morte no mundo e, entre a população idosa, este risco se eleva devido aos seus hábitos de vida e que têm como fatores de risco o sedentarismo, má alimentação, tabagismo e a não realização de atividades físicas, além de fatores metabólicos, como a hipertensão arterial, o sobrepeso/obesidade, diabetes e dislipidemia.

Notou-se, no decorrer dos primeiros encontros, a partir da análise da realidade exposta pelos idosos, que eles tinham dúvidas relacionadas a prevenção, tratamento e controle de enfermidades presentes em seu cotidiano. Outras questões observadas foram sobre conhecimento a respeito do autocuidado, ao processo de envelhecimento, aos seus direitos na sociedade, a prática de atividade física, aos hábitos alimentares saudáveis entre outros. Estas mesmas demandas foram observadas em um estudo envolvendo um projeto de extensão para idosos em João Pessoa, ressaltando a importância de iniciativas como esta relatada que aproximam o saber cientifico e popular às comunidades (Silva et al., 2017; Martins et al., 2019; Coelho et al., 2019). 
Diante das propostas levantadas pelos idosos, a equipe do projeto se propunha trazer a cada encontro uma temática diferente e relevante para o público alvo, abrangendo temas debatidos em âmbito regional, nacional e internacional, como por exemplo, o mês de outubro considerado como o "Mês do Idoso". Contou-se com a contribuição de profissionais de diversas áreas para explanar os temas abordados, de forma a estreitar relações entre profissionais e usuários (Carvalho \& Montenegro, 2012; Pitz \& Matsuchita, 2015).

Para tornar as atividades mais lúdicas, utilizou-se metodologias ativas, a saber: pinturas, recorte e colagem, danças, cartas, roda de conversa, folhetos, passeios a pontos turísticos da cidade, todos com o intuito de estimular funções cognitivas, físicas e interações sociais. A caracterização do espaço voltado para a temática abordada ou datas comemorativas, tornava o ambiente mais alegre, descontraído e despertava a curiosidade para as atividades que seriam desenvolvidas naquele encontro (Freire et al., 2017; Silva et al., 2017).

As particularidades do grupo eram sempre observadas e valorizadas pelos colaboradores, e com isso, tentava-se intervir em seus hábitos de vida através da exposição de variadas temáticas relacionadas ao seu cotidiano, em consonância com as principais políticas voltadas à pessoa idosa, não obstante, o projeto se propunha a atender suas necessidades, proporcionando vivências, interações e trocas de informações com outras pessoas (Cachioni et al., 2015).

Todas as atividades propostas pelo projeto serviram para que se conseguisse a confiança dos idosos, para assim possibilitar manutenção de vínculo. Observou-se boa interação do grupo com os colaboradores do projeto, pois constantemente participam ativamente do que era oferecido, interagiam e socializavam com os envolvidos. Essa metodologia ativa tinha como intuito dinamizar o processo ensino-aprendizagem, para que se pudesse obter uma efetiva comunicação e como resultado uma transformação em suas realidades, proporcionando uma manutenção da qualidade de vida desses idosos (Cachioni et al., 2015; Martins et al., 2019; Kahl et al., 2018).

Os impactos sociais fomentados em ações de grupos de extensão universitária, que têm como público alvo a população idosa, são notados a partir de comentários positivos acerca das atividades realizadas e como isso o ajudou de alguma forma na transformação de sua realidade. Os mesmos resultados foram destacados em um estudo da Universidade do Vale do Taquari acerca dos impactos de um projeto de extensão voltado para a qualidade de vida dos idosos, o qual foi avaliado como ótimo por mais de 78\% dos idosos participantes (Martinez et al., 2017)

As ações de promoção à saúde do projeto de extensão são ferramentas importantes no processo de minimizar ou diminuir danos à saúde, pois uma vez vinculada ao saber científico, são capazes de transformar a realidade do indivíduo em diversos aspectos. A exemplo da eficácia desse tipo de trabalho extracurricular, cita-se um estudo realizado por acadêmicos extensionistas de um projeto que tem como público alvo idosos do município de Porto Alegre, em que foi demonstrado que a prática de atividades físicas em grupo é uma forma de prevenção de depressão na terceira idade, logo, os idosos que fazem parte do grupo, tiveram benefícios à sua saúde mediante ações do projeto (Possamai et al., 2017)

\subsection{A extensão universitária e sua relação com o processo formativo profissional em gerontogeriatria}

A extensão universitária é uma atividade de grande relevância na formação acadêmica, profissional e humana de um indivíduo, pois abrange aspectos essenciais como educação, cultura, ciência, política e interdisciplinaridade. Esta propicia a construção de um vínculo entre a universidade e a sociedade, assim como promove uma colaboração recíproca de conhecimentos e experiências. No mais, a extensão deve ofertar serviço a todas as classes sociais, destacando as classes menos favorecidas, visto que estas têm menos acesso às informações, cumprindo assim o compromisso social da universidade e a transformação da realidade de maneira consciente e responsável (Medeiros, 2018).

Os projetos destinados às ações educativas estreitam a relação da comunidade acadêmica com a população, levando informações embasadas cientificamente, contribuindo para a formação de indivíduos conscientes do seu papel no bem-estar, 
assim como influenciadores nas suas comunidades para mudanças de hábitos e atitudes que afetam a coletividade. Educação em saúde torna-se uma ferramenta para o cuidar, em que o profissional da saúde pode utilizar desse instrumento para sensibilizar o seu cliente, familiares e cuidadores a uma melhor adesão a tratamentos e outros comportamentos que resultem em qualidade de vida (Freire et al., 2017; Guedes, 2014; Pitz \& Matsuchita, 2015; Coelho et al., 2019).

A Promoção da Saúde, por meio da educação, é uma abordagem amplamente discutida atualmente, promovendo melhora das condições de vida sendo papel de todas as esferas governamentais, porém para que seja cumprida, deve-se haver harmonia entre todas as partes envolvidas nesse processo. Trata-se então de uma estratégia para reduzir parte dos vários problemas de saúde que atingem as populações, pois prioriza a melhoria da qualidade de vida dos cidadãos, buscando o distanciamento do modelo biomédico (Pitz \& Matsuchita, 2015; Coelho et al., 2019).

Através das vivencias oportunizadas, os discentes de graduação, pós graduação e docentes tiveram a possibilidade de estar mais próximo da realidade da população, visualizar as reais demandas, compreendendo a importância do enfermeiro como agente promotor da saúde no SUS, corroborando para tornar essas experiências mecanismos de transformação na vida desses e profissionais e futuros profissionais (Cachioni et al., 2015; Coelho et al., 2019).

Nesta perspectiva, o profissional Enfermeiro ao imergir na realidade da população a qual ele presta seus cuidados, torna evidente sua relevância no sistema de saúde, principalmente, quando se intervém na realidade de pessoas idosas. Outrossim, o profissional que age desta forma cria vínculo com estas pessoas e seus cuidados são prestados de forma integral, levando em consideração todos os aspectos de seus usuários (Coelho et al., 2019; Kahl et al., 2018).

Nessa conjuntura, inserir o estudante no contexto de cuidado ao idoso é imprescindível, já que tendência mundial paira sobre o aumento da expectativa de vida e se percebe a necessidade de compreensão dos fatores relacionados ao envelhecimento humano. Além disso, o futuro profissional estará mais sensibilizado quanto a humanização, considerando essa fase como uma etapa normal da vida, buscando o envelhecimento saudável, através da prevenção de doenças, manutenção da saúde, independência e autonomia dos idosos. Então, a extensão promove esse contato mais minucioso e facilita a abordagem desses indivíduos tanto durante a graduação quanto no âmbito profissional (Lucena, 2017).

Portanto, para que essas ações possam ser efetivas, faz-se necessário a utilização de metodologias ativas em suas abordagens - quer seja em consultas individualizadas ou em ações educativas em saúde na comunidade -, estimulando os idosos e, assim, proporcionando a eles pensamentos críticos, que os auxiliem em sua autonomia na sociedade e que sejam os protagonistas de suas vidas e escolhas (Beserra et al., 2011; Martins et al., 2019).

\section{Considerações Finais}

Tendo em vista as especificidades que abarcam o processo de envelhecimento, o desenvolvimento de atividades extensionistas mostram-se como uma valiosa ferramenta para promoção do envelhecimento ativo e saudável da população assistida. Através da formação de grupos, os idosos são estimulados ao processo de socialização e, consequentemente, ao fortalecimento de vínculos, propiciando a criação de um ambiente acolhedor e seguro.

O processo de acolhimento desenvolvido nas atividades de extensão é vital para o sucesso das atividades, pois é o momento em que o idoso é cativado e posteriormente mais propenso a ter uma participação mais ativa. Esse aspecto contribui para um eficaz processo de aprendizado, os tornando indivíduos mais críticos com a sua realidade, tendo em vista aspectos sociais, fisiológicos e psicológicos. Idosos que possuem mais acesso às práticas e conhecimentos confiáveis, tornam-se mais conscientes perante seus direitos e deveres, bem como independentes na busca de uma qualidade de vida.

Ademais, nota-se a importância da extensão universitária na formação de profissionais de saúde, pois esta promove uma aproximação com a comunidade, gerando assim a construção de uma visão mais humanizada quanto a população idosa, 
oferecendo uma linha de cuidados mais integralizada, objetivando o envelhecimento ativo e saudável, Bem como, colabora na formação do senso de percepção dos condicionadores de saúde para além do biológico, promovendo assim um senso de cidadania nos indivíduos e agentes formadores da saúde pública.

Nesta perspectiva, salienta-se a necessidade do aprimoramento de estudos relacionados a extensão universitária como uma prática de abordagem de educação e promoção a saúde na prestação de cuidados de enfermagem às pessoas idosas, outrossim, estudos futuros devem reforçar a importância da ampliação do processo de formação acadêmica, no que diz respeito a inserção dos discentes, docentes e profissionais dos serviços de saúde na integração ensino-aprendizagem por meio das ações extensionistas. Com isso, fomenta-se o protagonismo e fortalecimento dos serviços de atenção à saúde da pessoa idosa e agrega na produção de tecnologias para atender e compreender as particularidades do gerenciamento do cuidado no processo de envelhecimento.

\section{Agradecimentos}

À Universidade Federal do Pará por meio da Pró-Reitoria de Extensão pelas concessões de bolsas de extensão (PIBEX), aos idosos do Projeto Idoso Saudável, pelo tempo, disposição e sabedoria doados no decorrer das nossas atividades.

\section{Referências}

Alves, V. M. C., Soares, V. N., Oliveira, D. V. D., \& Fernandes, P. T. (2020). Sociodemographic and psychological variables, physical activity and quality of life in elderly at Unati Campinas, São Paulo. Fisioterapia em Movimento, 33. http://dx.doi.org/10.1590/1980-5918.033.ao10.

Benedetti, T. R. B., Mazo, G. Z., \& Borges, L. J. (2012). Condições de saúde e nível de atividade física em idosos participantes e não participantes de grupos de convivência de Florianópolis. Ciência \& Saúde Coletiva, 17, 2087-2093. https://www.scielosp.org/article/csc/2012.v17n8/2087-2093/pt/.

Bergman, Y. S., \& Bodner, E. (2020). Age is not just a number: age awareness, subjective nearness-to-death, self-esteem, and depressive symptoms among older adults. Aging \& mental health, 24(6), 906-913. https://doi.org/10.1080/13607863.2019.1566815.

Beserra, Eveline Pinheiro, Torres, Cibele Almeida, Pinheiro, Patrícia Neyva Costa, Alves, Maria Dalva Santos, \& Barroso, Maria Grasiela Teixeira. (2011). Pedagogia freireana como método de prevenção de doenças. Ciência \& Saúde Coletiva, 16(1), 1563-1570. https://dx.doi.org/10.1590/S141381232011000700092 .

Cachioni, Meire, Ordonez, Tiago Nascimento, Batistoni, Samila Sathler Tavares, \& Lima-Silva, Thaís Bento. (2015). Metodologias e Estratégias Pedagógicas utilizadas por Educadores de uma Universidade Aberta à Terceira Idade. Educação \& Realidade, 40(1), 81-103. https://dx.doi.org/10.1590/2175-623645741

de Carvalho, B. G. C., \& Montenegro, L. C. (2012). Metodologias de comunicação no processo de educação em saúde. Revista de Enfermagem do CentroOeste Mineiro. https://doi.org/10.19175/recom.v0i0.148.

Coelho, A. A. R., dos Santos, A. D. F., da Silva, L. G., Machado, M. D. F. A. S., de Araújo Alves, D., Lemos, I. C. S., \& Bezerra, I. M. P. (2019). Health Education for Elderly: from Organization to Practice of the Nurse. Journal of Health Sciences, 21(2), 170-174. https://doi.org/10.17921/24478938.2019v21n2p170-174.

Freire, M. T. J., Brandão, M. G. S. A., de Mesquita Braga, M., Silva, R. A., \& Freitas, C. A. S. L. (2018). Grupos de idosos como estratégia de promoção da saúde: Relato de experiência. Essentia-Revista de Cultura, Ciência e Tecnologia da UVA, 19(1). https://essentia.uvanet.br/index.php/ESSENTIA/article/view/154.

Guedes, Simoni Lahud. (2014). Vidas Revisitadas: A reconstrução do passado de idosos em programas gerontológicos. Sociologia \& Antropologia, 4(1), 233255. https://doi.org/10.1590/2238-38752014v4110.

Kahl, C., Meirelles, B., Lanzoni, G., Koerich, C., \& Cunha, K. (2018). Actions and interactions in clinical nursing practice in Primary Health Care. Ações e interações na prática clínica do enfermeiro na Atenção Primária à Saúde. Revista da Escola de Enfermagem da USP, 52, e03327. https://doi.org/10.1590/s1980-220x2017025503327.

de Lucena, K. D. T., Deininger, L. D. S. C., de Brito, A. L. S., Nogueira, A. P. F., Neves Filho, G. H. C., Menezes, I. O., \& Viegas, M. E. P. (2017). University Extension of Elderly Health and Potency for Medical Training. International Archives of Medicine, 10. https://doi.org/10.3823/2445.

Mallmann, D. G., Galindo Neto, N. M., Sousa, J. D. C., \& Vasconcelos, E. M. R. D. (2015). Educação em saúde como principal alternativa para promover a saúde do idoso. Ciência \& Saúde Coletiva, 20, 1763-1772. https://doi.org/10.1590/1413-81232015206.02382014.

Martinez, B. B., Thomé, A. L. E., Kerkhoff, A. C., \& Brod, A. (2017). O impacto do projeto ações sociais e de saúde em gerontologia/Univates (RS) para idoso. Extensio: Revista Eletrônica de Extensão, 14(27), 34-40. http://dx.doi.org/10.5007/1807-0221.2017v14n27p34. 
Martins, Rita de Cassia Cabral de Campos, Casetto, Sidnei José, \& Guerra, Ricardo Luís Fernandes. (2019). Mudanças na qualidade de vida: a experiência de idosas em uma universidade aberta à terceira idade. Revista Brasileira de Geriatria e Gerontologia, 22(1), e180167. Epub June 10, 2019. https://dx.doi.org/10.1590/1981-22562019022.180167.

Medeiros, F. D. A. L., Nóbrega, M. M. D. L., Medeiros, A. C. T. D., Bittencourt, K. G. D., \& Araújo, G. D. L. (2015). Contextualização do envelhecimento saudável na produção científica brasileira. Rev. enferm. UFPE on line, 985-993. https://periodicos.ufpe.br/revist as/revistaenfermagem/article/download/10424/11214.

Medeiros, I. de M. (2018). A criação e implementação do programa de extensão AFRID da Faculdade de Educação Física da Universidade Federal de Uberlândia (1989-2014). Revista Em Extensão, 17(1), 9-39. https://doi.org/10.14393/REE-v17n12018-art01.

Morley, J. E., \& Malmstrom, T. K. (2013). Frailty, sarcopenia, and hormones. Endocrinology and Metabolism Clinics, 42(2), 391-405. https://doi.org/10.1016/j.ecl.2013.02.006.

de Fáveri Pitz, A., \& Matsuchita, H. L. P. (2015). Importância da Educação em Saúde na Terceira Idade. UNICIÊNCIAS, 19(2). https://revista.pgsskroton.com/index.php/uniciencias/article/download/3595/3126.

Possamai, V. D., Cardoso, P., de Vargas, A. S. R., Dutra, N. S., \& Gonçalves, A. K. (2017). Sintomatologia depressiva em idosos e a influência da atividade física em um programa de extensão. Extensio: Revista Eletrônica de Extensão, 14(27), 93-100. http://dx.doi.org/10.5007/807-0221.2017v14n27p93.

Pereira, A. S., Shitsuka, D. M., Parreira, F. J., Shitsuka, R. (2018). Metodologia da pesquisa científica. Núcleo de Tecnologia Educacional. UFSM. https://repositorio.ufsm.br/bitstream/handle/1/15824/Lic_Computacao_Metodologia-Pesquisa-Cientifica.pdf?sequence=1.

Santos, B. R., Pavarini, S. C. I., Brigola, A. G., Orlandi, F. de S., \& Inouye, K. (2014). Factors associated with quality of life in elderly undertaking literacy programs. Dementia \& Neuropsychologia, 8(2), 169-174. https://doi.org/10.1590/S1980-57642014DN82000013.

Silva, W., De Lucena, A. L. R., Araújo, M. J. A., Januário, D. C., Vieira, K. F. L., \& Costa, R. D. R. A. B. (2017). Ações educativas vivenciadas com idosos: $\mathrm{Um}$ relato de experiência. Revista de Ciências da Saúde Nova Esperança, 15(3), 31-36. http://revista.facene.com.br/inde x.php/revistane/article/download/67/73.

Síntese de indicadores sociais: uma análise das condições de vida da população brasileira: 2016 / IBGE, Coordenação de População e Indicadores Sociais. Rio de Janeiro: IBGE, 2016

Tessari, A. A., Giehl, M. W. C., Schneider, I. J. C., \& González-Chica, D. A. (2016). Anthropometric measures change and quality of life in elderly people: a longitudinal population-based study in Southern Brazil. Quality of Life Research, 25(12), 3057-3066. https://doi.org/10.1007/s11136-016-1330-6.

United Nations, Department of Economic and Social Affairs, Population Division (2020). World Population Ageing 2019 (ST/ESA/SER.A/444). (NY): United Nations. https://www.un.org/en/development/desa/population/publications/pdf/ageing/WorldPopulationAgeing2019-Report.pdf.

World Health Organization (WHO). Noncomunicable diseases. http://www.who.int/mediacentre/factsheets/fs355/en/. 